\title{
DYNAMIC CHANGE OF NT- PROBNP IN PATIENTS WITH ST-ELEVATION MYOCARDIAL INFARCTION TREATED WITH PRIMARY CORONARY INTERVENTION
}

\author{
Javor Kashlov ${ }^{1,2}$, Veselin Valkov ${ }^{1,4}$, Jordanka Doneva ${ }^{1,2}$, Ivan Donev ${ }^{1,3}$, \\ Arpine Kirkorova ${ }^{1,2}$, Galina Arabadzhieva ${ }^{4}$, Lilia Ivanova ${ }^{5}$, Nikolay Conev $^{1,3}$, \\ Borislav Ivanov ${ }^{7}$, Temenuzhka Radeva ${ }^{6}$, Zhaneta Georgieva ${ }^{1,2}$ \\ ${ }^{1}$ Department of Propedeutics of Internal Medicine, Medical University of Varna, Bulgaria \\ ${ }^{2}$ Department of Internal Medicine, St. Marina University Hospital, Varna, Bulgaria \\ ${ }^{3}$ Clinic of Medical Oncology, St. Marina University Hospital, Varna, Bulgaria \\ ${ }^{4}$ Department of Cardiology, St. Marina University Hospital, Varna, Bulgaria \\ ${ }^{5}$ Department of Microbiology and Virology, Medical University of Varna \\ ${ }^{6}$ Department of Radiation Oncology and Imaging Diagnostics, \\ St. Marina University Hospital, Varna Bulgaria; \\ ${ }^{7}$ Department of Clinical Medical Sciences, Medical University of Varna, Bulgaria
}

\begin{abstract}
In the recent years important biomarkers have emerged as tools for diagnosis and risk stratification in cardiovascular diseases. Such markers are B-type natriuretic peptide (BNP) and N-terminal B-type natriuretic peptide (NT-proBNP).

Peripheral blood for the serum levels of NT-proBNP was taken from patients with STEMI before PCI and 2448 hours after the onset of the symptoms of myocardial infarction. Three of the samples from all 53 turned out to be positive to NT-proBNP concentration on Day 0. On day 1 there was a significant elevation of the positive samples 11 from 53 patients $(20.7 \%)(p=0.01)$. All patients with STEMI and elevated serum levels of NT-proBNP have left ventricular ejection fraction $<50 \%$.

Our results imply that NT- proBNP level and its increase in the serum may be used as a biomarker for the severity of the ischemic heart disease.
\end{abstract}

Keywords: myocardial infarction, ejection fraction, NT-proBNP, percutaneous coronary intervention

\section{INTRODUCTION}

The prevalence of acute and chronic ischemic heart disease and heart failure reaches up to $8 \%$ in the Western countries. Heart failure and acute myocardial infarction (AMI) remain the leading causes of morbidity and mortality in the developed countries (1).

It has been proven, that the most effective treatment method of one of the forms of ischemic heart diseases - AMI is immediate reperfusion achieved through percutaneous coronary intervention (PCI). This method allows early restoration of blood flow in the infarct- related artery (IRA) and is the principal mechanism by which reperfusion therapy improves the outcome in patients with AMI $(2,3)$.

Received: November 11, 2016

Accepted: December 19, 2016 
Nevertheless, patients with AMI are found to have a $10 \%$ higher risk of death and $25 \%$ higher risk of developing heart failure within the first year of the ischemic attack (4).

In the recent years important biomarkers have emerged as tools for diagnosis and risk stratification in cardiovascular diseases. Such markers are B-type natriuretic peptide (BNP) and N-terminal B-type natriuretic peptide (NT-proBNP). Both molecules are constantly released and can be detected in blood. The main stimulus for their secretion is myocardial wall stress. These peptides are released predominantly from the myocardium in response to myocyte stretch.They are produced from a prohormone, which is split into BNP and NT-proBNP (5). According to the European Society of Cardiology they are recommended to be measured in diagnosing chronic heart failure (6).

Several reports, however, indicate that BNP and NT-proBNP were increased in the serum of patients with acute myocardial infarction (AMI) and showed usefulness in detecting left ventricular dysfunction (7-11).

The goal of our study was to examine the predicitive value of the serum levels of NT-pro BNP in patients with ST-elevation myocardial infarction (STEMI) treated with percutaneous coronary intervention (PCI) after a median follow-up of 12 months.

\section{MATERIALS AND METHODS}

\section{Ethics Statement}

All procedures were approved by the Scientific Research Ethics Commitee of the Medical University of Varna.

The current study was conducted at the St. Marina University Hospital, Varna after an informed consent form (ICF) from all study participants.

\section{Patient Selection}

From October, 2014 to April, 2016, blood samples were taken from 53 patients with STEMI .

Patients were treated with percutaneous coronary intervention (PCI) within the first 12 hours of the onset of clinical manifestation and had postprocedure Thrombolysis in Myocardial Infarction (TIMI) flow grade 3.

Serum levels of NT-proBNP were measured upon admission on Day 0 ( $<12$ hours from the on- set of the symptoms) and on Day 1 (24-48 hours after the onset of symptoms of myocardial infarction). The time of the onset of symptoms and the first blood test is less than 1 hour for 2 patients (3.9\%), 1-3 hours - 19 patients (37.2\%), 3-6 hours - 20 patients (39.2\%) and 6-12 hours - 12 patients (23.5\%).

Inclusion criteria for the patients with myocardial infarction in the current study were: chest pain with significant (minimum $2 \mathrm{~mm}$ ) ST-segment elevation in at least 2 contiguous electrocardiogram (ECG) leads and/or a significant increase in cardiac markers (troponin $\mathrm{I}>0.2 \mathrm{ng} / \mathrm{ml}$ ), $<12$ hours after the onset of symptoms to primary PCI, all patients underwent cardiac catheterization and were treated with primary coronary angioplasty and stent replacement. Exclusion criteria were clinical or laboratory evidence of current infection, other inflammatory or oncologic disease.

\section{Echocardiography}

Left ventricular ejection fraction (LVEF) was evaluated and recorded by a biplane method of disks (Simpson's rule) in accordance with the European Association of Cardiovascular Imaging.

Measurement of the Levels of NT-proBNP in the Serum

Peripheral blood sample from the patients with STEMI was taken before PCI and again 24-48 h after the onset of symptoms. The amount of every blood sample was $8 \mathrm{ml}$ divided into 2 tubes and within 15 minutes centrifuged at 2500 for $20 \mathrm{~min}$. Serum was frozen and stored at $80 \mathrm{C}$. Concentration of NTproBNP was determined using an ELISA (CUSABIO, Wuhan, China) according to the manufacturer's instructions.

\section{Statistical Analysis}

Statistical analysis was carried out by SPSS Statistics v.23 using descriptive statistics.

Categorial features were summarized with frequencies and percentages. Variables were reported as mean \pm standard deviation (SD). Wilcoxon paired test was used.

\section{RESULTS}

Demographic Characteristics and Follow-Up of the Patients

In the group of patients with STEMI men were $56.6 \%$, while women $-43.4 \%$. The average age of the 
Javor Kashlov, Veselin Valkov, Jordanka Doneva et al.

patients with STEMI was $63.9 \pm 12.9$ with the minimum age being 29 and the maximum - 85 years.

Demographic characteristics of the patients with STEMI are shown in Table 1.

Table 1. Baseline characteristics of STEMI patients (ST-elevation myocardial infarction)

\begin{tabular}{lccc} 
Demographic parameters & STEMI & Significance \\
Age, yrs. & $63.9 \pm 12.9$ & $p=0.32$ \\
Male, \% & 57.4 & $p=0.43$ \\
Hypertension, \% & 100 & - \\
Diabetes, \% & 15.7 & $p=0.68$ \\
Hypercholesterolemia, \% & 70.2 & $p=0.13$ \\
\hline
\end{tabular}

Median follow-up of the STEMI patients was 12 months. After the follow-up, 5 STEMI patients died from cardiac causes and 4 STEMI patients were further hospitalized due to symptoms of heart failure.

Serum Levels of NT- proBNP in Patients with STEMI

Peripheral blood for the serum levels of NTproBNP was taken from patients with STEMI before PCI and 24-48 hours after the onset of the symptoms of myocardial infarction. Three of the samples from all 53 turned out to be positive to NT-proBNP concentration on Day 0. On Day 1 there was a significant elevation of the positive samples (Figure 1) - 11 from 53 patients $(20.7 \%)(\mathrm{p}=0.01)$. From those 11 patients, 6 were with two -vessel coronary artery disease, 2 patients - with three vessel coronary artery disease and 3 patients with single-vessel coronary artery disease. Three of these patients died during follow-up.

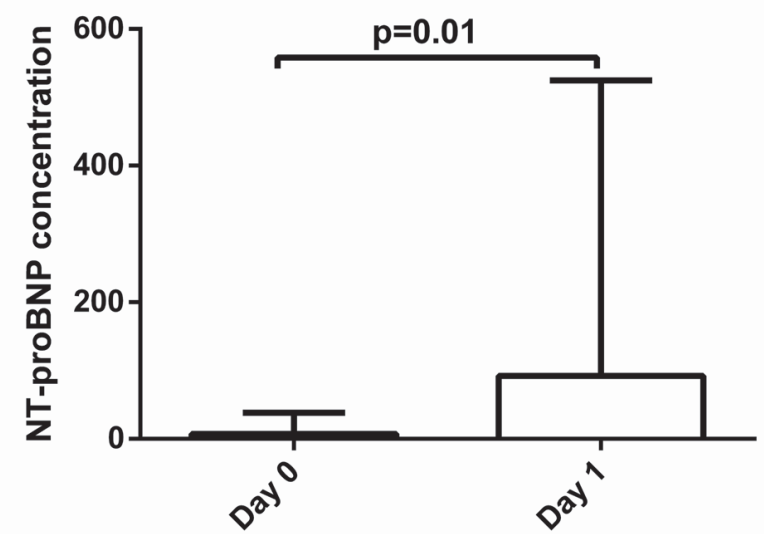

Fig. 1. Serum levels of NT-proBNP in patients with ST-elevation myocardial infarction (STEMI) on Day 0 and Day 1
Figure 1 shows the serum levels of NT-proBNP in patients with ST-elevation myocardial infarction (STEMI) on Day 0 and Day 1. A value of $\mathrm{p}<0.05$ is considered statistically relevant.

All patients with STEMI and elevated serum levels of NT- proBNP have left ventricular ejection fraction $<50 \%$. There is a significant correlation between the serum levels of NT-proBNP and the serum levels of RIPK3 - a marker for necroptosis $(r=0.629$, $\mathrm{p}=0.038)$ and a weak correlation between NT-proB$\mathrm{NP}$ and Troponin I $(\mathrm{r}=0.296, \mathrm{p}=37)$.

\section{DISCUSSION}

In our study we confirm the results that patients with STEMI 24-48 h after the onset of the symptoms and ejection fraction $(\mathrm{EF}<50 \%)$ showed increased serum levels of NT-proBNP. According to the European Society of Cardiology the N-terminal part of the pro - B type natriuretic peptide (NT-proBNP) is recommended to be measured in diagnosing heart failure (6). NT-proBNP plasma concentrations also typically increased in patients with asymptomatic or symptomatic left ventricular dysfunction are also associated with coronary artery disease and myocardial ischemia (12). NT-proBNP is mainly released from myocytes in the cardiac ventricles as a result of wall tension and stretch in response to stimuli such as ischemia or cytokine secretion. The role and participation of this molecule in different cardiovascular diseases has been discussed well (13).

In a study measuring the concentration of NTproBNP in patients with normal or slightly decreased EF this marker was shown to correlate with left ventricular systolic impairment and left ventricular systolic ejection fraction $<40 \%$.

In a study that we performed we also examined RIPK3 - a novel regulator of programmed cell death in healthy people and its correlation with NT proBNP (14). This study included the same 53 patients with STEMI. Programmed necrosis mediated by RIPK 3 - a regulator of programmed cell death has recently been defined as a novel mechanism of cell death with major functional importance in several organs, including the heart (15). RIPK3 is also responsible for generation of reactive oxygen species, which is supposed to be another mechanism for organ damage due to myocardial ischemia (16). Our results suggest that serum level of RIPK3 is significant- 
ly increased 24 hours after the onset of symptoms. There is a strong correlation between the serum levels of RIPK3 and NT-proBNP.

Serum levels of NT-proBNP are increased after 24-48 hours after the onset of symptoms of myocardial infarction and may define the degree of myocyte injury. Furthermore, our results imply that the NTproBNP level and its increase in the serum may be used as a biomarker for the severity of the ischemic heart disease.

New studies, however, are necessary to define the exact role of NT-proBNP for risk stratification and prognosis in patients with STEMI.

\section{CONCLUSION}

In summary our results suggest that patients with STEMI show increased serum levels of NTproBNP 24-48 hours after the onset of clinical manifestation of myocardial infarction. NT-proBNP may have the potential as a new biomarker to evaluate the prognosis in patients with STEMI.

\section{REFERENCES}

1. Gaziano, T., et al., Cardiovascular Disease, in Disease Control Priorities in Developing Countries, D.T. Jamison, et al., Editors. 2006: Washington (DC).

2. Andersen, H.R., et al., A comparison of coronary angioplasty with fibrinolytic therapy in acute myocardial infarction. N Engl J Med, 2003. 349(8): p. 733-42.

3. Boden, W.E., K. Eagle, and C.B. Granger, Reperfusion strategies in acute ST-segment elevation myocardial infarction: a comprehensive review of contemporary management options. J Am Coll Cardiol, 2007. 50(10): p. 917-29.

4. Keeley, E.C., J.A. Boura, and C.L. Grines, Primary angioplasty versus intravenous thrombolytic therapy for acute myocardial infarction: a quantitative review of 23 randomised trials. Lancet, 2003. 361(9351): p. 13-20.

5. Morita, E., et al., Increased plasma levels of brain natriuretic peptide in patients with acute myocardial infarction. Circulation, 1993. 88(1): p. 82-91.

6. Swedberg, K., et al., Guidelines for the diagnosis and treatment of chronic heart failure: executive summary (update 2005): The Task Force for the Diagnosis and Treatment of Chronic Heart Failure of the European Society of Cardiology. Eur Heart J, 2005. 26(11): p. 1115-40.

7. Nagaya, N., et al., Plasma brain natriuretic peptide is a biochemical marker for the prediction of progressive ventricular remodeling after acute myocardial infarction. Am Heart J, 1998. 135(1): p. 21-8.

8. Talwar, S., et al., Profile of plasma N-terminal proBNP following acute myocardial infarction, correlation with left ventricular systolic dysfunction. Eur Heart J, 2000. 21(18): p. 1514-21.

9. Groenning, B.A., et al., Detection of left ventricular enlargement and impaired systolic function with plasma N-terminal pro brain natriuretic peptide concentrations. Am Heart J, 2002. 143(5): p. 923-9.

10. Galvani, M., et al., N-terminal pro-brain natriuretic peptide on admission has prognostic value across the whole spectrum of acute coronary syndromes. Circulation, 2004. 110(2): p. 128-34.

11. Bazzino, O., et al., Relative value of N-terminal probrain natriuretic peptide, TIMI risk score, ACC/ AHA prognostic classification and other risk markers in patients with non-ST-elevation acute coronary syndromes. Eur Heart J, 2004. 25(10): p. 859-66.

12. Kim, H., et al., Incremental prognostic value of Creactive protein and $\mathrm{N}$-terminal proB-type natriuretic peptide in acute coronary syndrome. Circ J, 2006. 70(11): p. 1379-84.

13. Szadkowska, I., et al., The relationship between early recanalization and serum NT-proBNP levels in patients with a first ST-segment elevation myocardial infarction treated with primary coronary angioplasty. Acta Cardiol, 2007. 62(5): p. 479-84.

14. Kashlov, J.K., et al., Serum levels of RIPK3 and troponin I as potential biomarkers for predicting impaired left ventricular function in patients with myocardial infarction with ST segment elevation and normal troponin I levels prior percutaneous coronary intervention. Biosci Trends, 2016. 10(4): p. 294-9.

15. Linkermann, A. and D.R. Green, Necroptosis. N Engl J Med, 2014. 370(5): p. 455-65.

16. Zhao, H., et al., Role of necroptosis in the pathogenesis of solid organ injury. Cell Death Dis, 2015. 6: p. e1975. 